\title{
Stress-Induced Effects by the Anodic Oxide in Ridge Waveguide Laser Diodes
}

\author{
M. Buda, G. Iordache, G. A. Acket, T. G. van de Roer, L. M. F. Kaufmann, B. H. van Roy, E. Smalbrugge, \\ I. Moerman, and C. Sys
}

\begin{abstract}
This paper studies, both theoretically and experimentally, stress-induced effects on the lateral far-field behavior for ridge-type semiconductor laser diodes where anodic oxide is used for the definition of the stripe width. These effects consist of antiguiding under the stripe region, and of two positive waveguiding features near the stripe edges. For low-threshold devices, these effects may be more important than thermal effects, depending on the stress in the oxide. They put a lower limit on the built-in index guiding to be introduced by lateral etch outside the ridge region in order to maintain fundamental mode operation for wider stripes. The magnitude of these effects may be as large as $\Delta n_{\mathrm{ef}}=1 \times 10^{-3}$. An analytical mathematical model is deduced for computing stresses and strains for a certain ridge-shaped interface which bounds the elastic medium.
\end{abstract}

Index Terms-(Injection) lasers, optical waveguides, semiconductor device modeling, semiconductor lasers, strain.

\section{INTRODUCTION}

$\mathbf{T}$ HIS PAPER is concerned with the study of stress-induced effects on the far field of semiconductor laser diode devices in the lateral direction; that is, corresponding to the distribution of the optical field in the direction perpendicular to the growth direction. For high-power operation, to avoid facet damage, a larger optical stripe width would be required. This may be achieved by using a smaller built-in index step, which suppresses higher order modes. There is another problem to be solved: how weak can the built-in index guiding be?

Especially for lower confinement structures, carrier-induced antiguiding is decreased and thermal and stress-induced effects become of importance in weak lateral waveguides. This paper aims to estimate the magnitude of the latter. Thermal effects can be estimated from large threshold devices, where they introduce a significant delay between the beginning of the electrical pulse and optical pulse, on the order of $1 \mu \mathrm{s}$, if the built-in index guiding is not enough to overcome them [1]. The magnitude of thermal waveguiding is evaluated to be $\Delta n_{\mathrm{eff}} \approx 10^{-3}$ at threshold, for a stripe width of $8 \mu \mathrm{m}$ and for a threshold current density of $2500 \mathrm{~A} / \mathrm{cm}^{2}$.

Manuscript received August 23, 1999; revised May 30, 2000

M. Buda, G. Iordache, G. A. Acket, T. G. van de Roer, L. M. F. Kaufmann, B. $\mathrm{H}$. van Roy, and E. Smalbrugge are with COBRA Interuniversity Research Institute on Communication Technology: Basic Research and Applications, Eindhoven University of Technology, 5600 MB Eindhoven, The Netherlands (e-mail: M.Buda@phys.tue.nl).

I. Moerman and C. Sys are with the Department of Information Technology (INTEC), University of Gent-IMEC, St. Pieternieuwstraat 41, B-9000 Gent, Belgium.

Publisher Item Identifier S 0018-9197(00)08140-9.
The influence of stresses introduced during processing in oxide-insulated laser diodes and related stress-induced changes of the refractive index, which depend on the thickness and on the shape of the oxide layer, was reported at the beginning of the development of laser diodes [2]-[4]. Their further study was cast aside because the built-in effective refractive index step in conventional devices exceeded the influence of this effect. It was noticed, however, that if the active region is too close to the etched surface, the performance of the laser diodes become poorer.

Recently, planar semiconductor lasers using the photoelastic waveguiding effect induced by a WNi stress layer were reported in [5]. Stress-induced lateral confinement of light in epitaxial $\mathrm{BaTiO}_{3}$ films was obtained by Barrios et al. [6]. It is also shown that a deposition process itself may introduce a significant intrinsic stress, in addition to the thermal stress due to the cooling from the temperature during deposition down to room temperature. The intrinsic stress may even dominate over the stress induced by the thermal-expansion mismatch, especially when a film is deposited at room temperature.

Experimentally, using spatially-resolved and polarization-resolved photoluminescence, strain was measured in GaAs- and InP-based semiconductor devices with a strain resolution better than $10^{-5}$ and a spatial resolution of about $1 \mu \mathrm{m}$ [7]-[9]. These measurements show that the strain fields in semiconductor laser diodes are related to both geometrical structure and processing conditions of the device. Metallized sharp edges, including dielectrics, such as the ridge and etched channels formed in ridge waveguide lasers, tend to have large strain fields associated with them. The strain due to soldering itself usually introduces a roughly constant strain distribution in the active region.

For our devices, anodic oxide is used to restrict the current injection outside the stripe width. We evaluate the stress in the anodic oxide and estimate its effects on the profile of the effective refractive index starting from the quasi-2-D model presented in [1].

\section{Modeling OF STRESS-INDUCED EFFECTS}

\section{A. Introduction}

Computation of elastic stresses and strains is more complicated for the GaAs/oxide system than for $\mathrm{Si} / \mathrm{SiO}_{2}$ system since it has an anisotropic elastic compliance tensor. Although the computational approach resulting from [10], that we use here, can also be used for the anisotropic case, in the following, an isotropic average value for Young's modulus $E_{e}$ and Poisson's ratio $\nu$ will be used, as in [2]. 


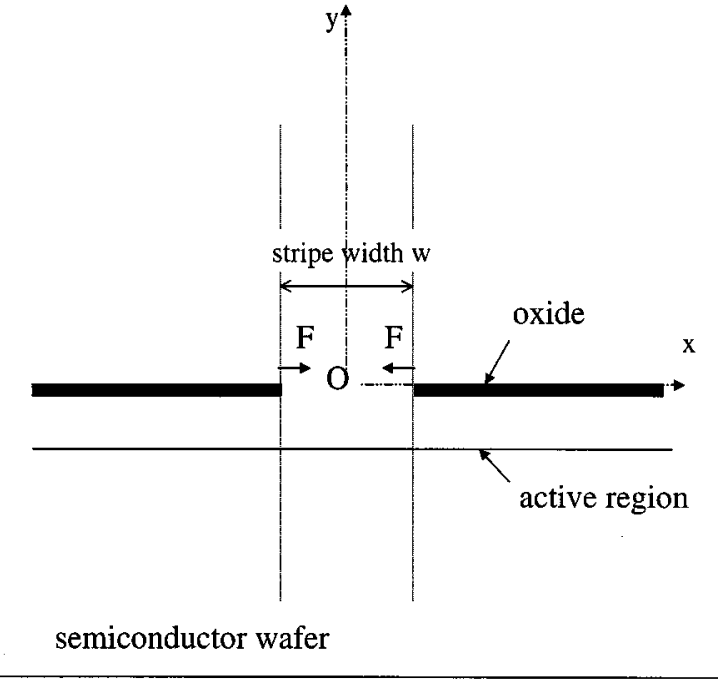

a)

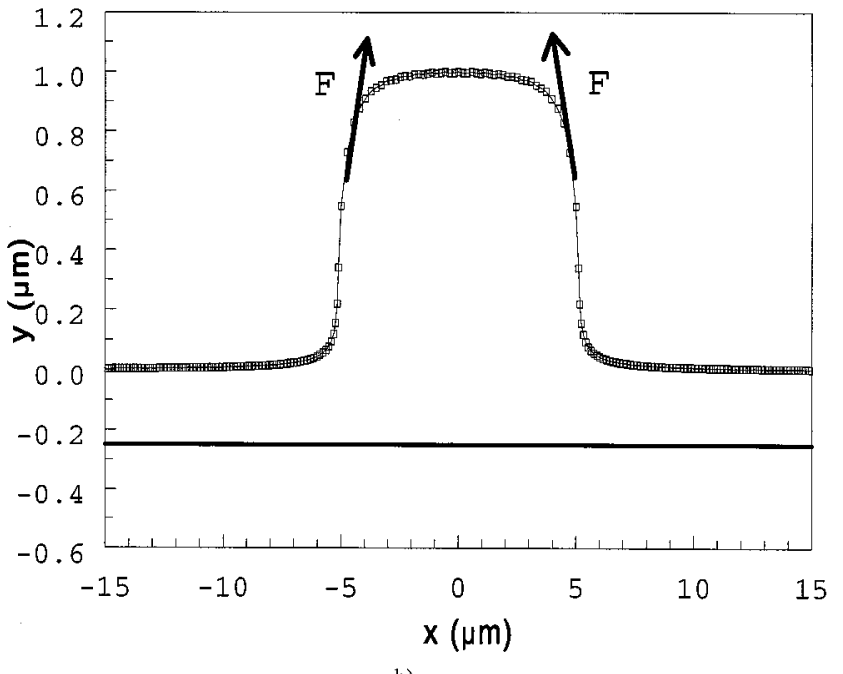

b)

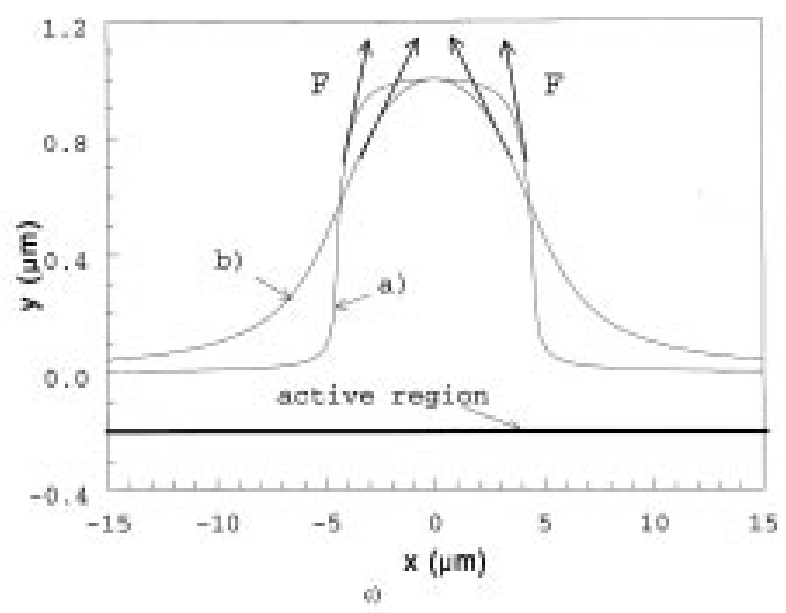

Fig. 1. (a) Half-plane approximation. (b) Ridge-shaped approximation. Parameters related to the Appendix are: $h=1 \mu \mathrm{m}, W=6.5 \mu \mathrm{m}, W_{f}=6 \mu \mathrm{m}$, $p=0.25 \mu \mathrm{m}$. (c) Ridge-shaped profiles. Parameters related to the Appendix are: (a) $h=1 \mu \mathrm{m}, W=6.5 \mu \mathrm{m}, W{ }_{f}=6 \mu \mathrm{m}, p=0.3 \mu \mathrm{m}$. (b) $h=1 \mu \mathrm{m}$, $W=6.5 \mu \mathrm{m}, W_{f}=6 \mu \mathrm{m}, p=2.5 \mu \mathrm{m}$.

For simple planar gain-guided oxide-defined stripe lasers, such as those used early in the history of semiconductor laser diodes, the simple case of a half-plane with two concentrated forces acting at the stripe edges can be considered. This is illustrated in Fig. 1(a). Next, ridge waveguides with a certain profile above the $0 x$ axis are analyzed, in order to simulate the case of ridge waveguide index-guided devices as in Fig. 1(b) and (c). A simplified shape of the ridge is considered to allow for analytic computations. For mathematical purposes, the function that describes the ridge shape and its derivative are considered to be continuous. The mathematical procedure for deriving stresses $\sigma_{x x}, \sigma_{y y}$ and the corresponding strains by $e_{x x}, e_{y y}$, and $e_{z z}$ is described in detail in the Appendix.

The solution of the problem for the half-plane and concentrated forces is well known from the literature. In particular, complex methods are very powerful, allowing one to obtain relatively easily an analytical solution. Using the methods from [10] for a concentrated force and extending to the case presented in
Fig. 1(a), one can obtain the stress and strain for the half-plane in a quite straightforward manner. We are looking for the plane stress problem, i.e., we assume that the $z$ component of the displacement is zero and that the $x$ and $y$ components depend only on $x$ and $y$ and not on $z$. We also assume that the boundary value of the stress tensor is given. The symmetric stress tensor for the plane problem has only three distinct terms: $\sigma_{x x}, \sigma_{x y}$, and $\sigma_{z z}$. For the ridge profile, a similar procedure is used, but considering a conformal transformation which relates points in the half-plane with the ridge-type shape (see Appendix).

The dielectric constant (and hence, the refractive index) of a crystal is dependent on the stress (or strain) in the crystal. This effect is called the photoelastic effect; it is similar to the electrooptic effect, which is the effect of an applied electric field on the dielectric constant of a crystal. In the following, the same constants as in [2] are used to compute the changes of the refractive index for the TE wave from the strain distribution. That 


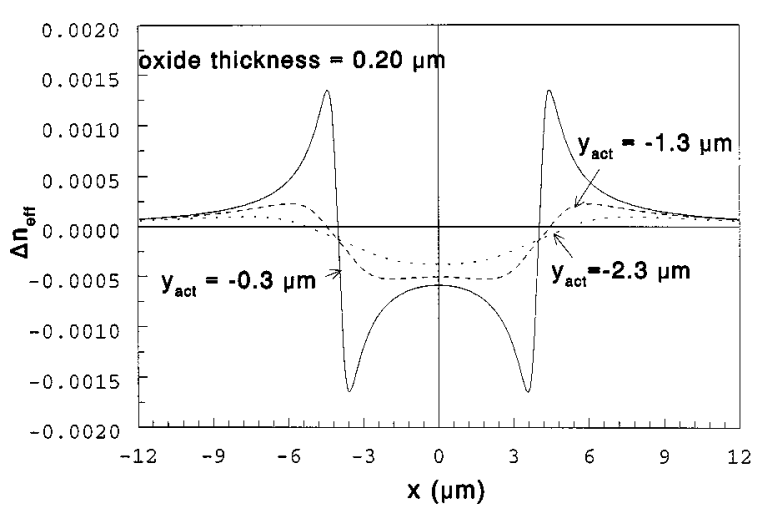

(a)

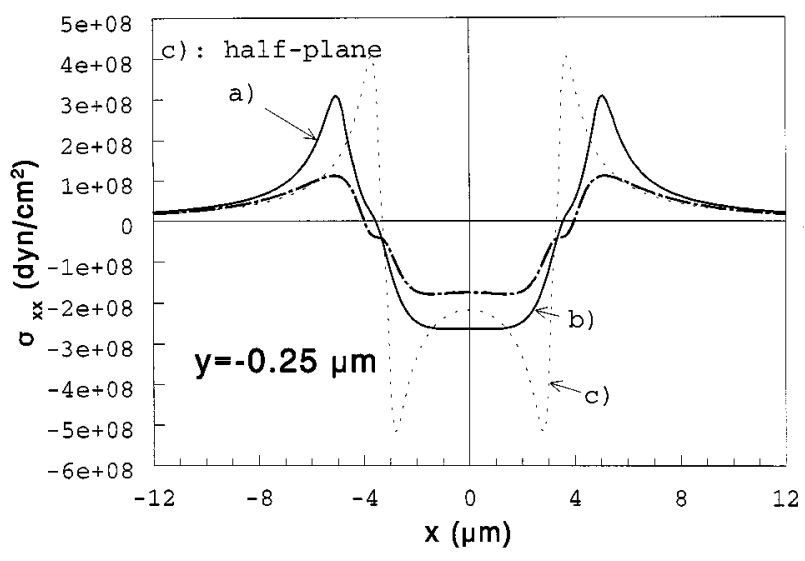

c)

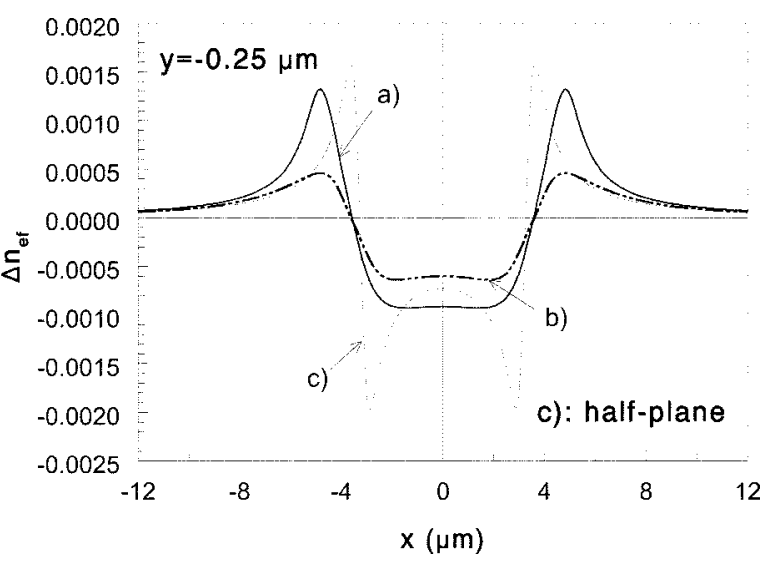

(b)

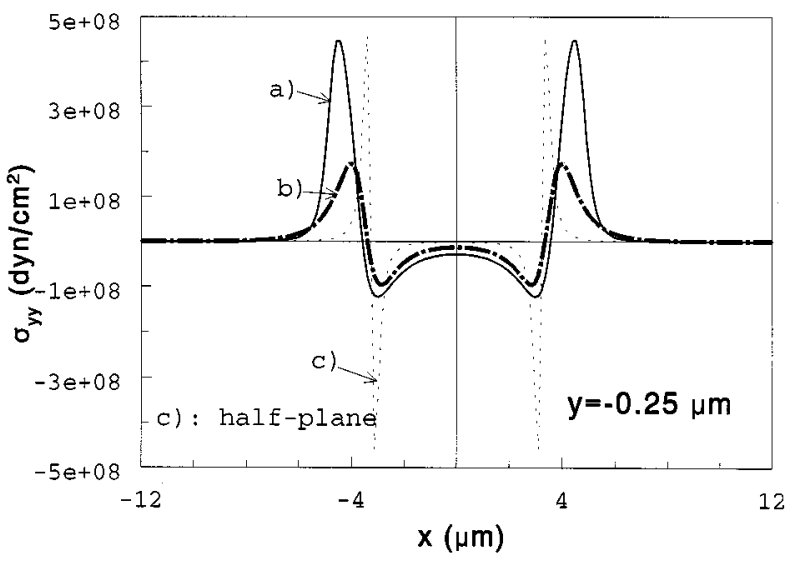

d)

Fig. 2. (a) Changes of the refractive index at different depths in the semiconductor material for the half-plane approximation. The stripe width is $8 \mu \mathrm{m}$. (b) Changes of the refractive index at $y=-0.25 \mu \mathrm{m}$ for the shaped ridge profiles presented in Fig. 1(c). The oxide thickness is $0.2 \mu \mathrm{m}$. (c) Stress dependence $\sigma_{x x}$ along the stripe width for the ridge profiles presented in Fig. 1(c). (d) Stress dependence $\sigma_{y y}$ along the stripe width for the ridge profiles presented in Fig. 1(c).

is, the change of the dielectric constant $\Delta \varepsilon_{x x}$ is given by

$$
\Delta \varepsilon_{x x}=-\varepsilon^{2} \cdot\left\{e_{x x} \cdot\left[\frac{\left(p_{11}+p_{12}\right)}{2}+p_{44}\right]+e_{z z} \cdot p_{12}\right\}
$$

where the photoelastic coefficients are $p_{11}=-0.165, p_{12}=$ -0.140 and $p_{44}=-0.072$.

\section{B. Computational Results}

The computational results for the case of the half plane are given in Fig. 2(a), which presents the change of the refractive index, for a stripe width of $8 \mu \mathrm{m}$, at different depths in the semiconductor material. First, we notice that the influence of the oxide induced stress is mainly antiguiding (its value in the middle of the stripe being of the order of $5 \times 10^{-4}$ ) and does not change significantly if the active region is placed at a depth of $1.3 \mu \mathrm{m}$ or $2.3 \mu \mathrm{m}$, for example. That is the case for gain-guided oxide-stripe devices. If the active region lies only $0.3-\mu \mathrm{m}$ below the $0 x$ axis, the picture is changed considerably. We see a strong perturbation at the stripe edges, which has the maximum amplitude value of $\Delta n_{\text {eff }}$ of about $1.5 \times 10^{-3}$, and also a slight waveguiding profile under the stripe. For our ridge waveguide devices, the active region is about $0.3-\mu \mathrm{m}$ below the etched material outside the stripe region, and about $1.3-\mu \mathrm{m}$ below the un- etched surface of the stripe region [see Fig. 1(b)]. Thus, we also considered the problem of a shaped ridge separately.

Fig. 2(b) presents the variation of the refractive index given by a stress distribution due to two concentrated forces [as in Fig. 1(c)], for a total stripe width of $8 \mu \mathrm{m}$ and different shapes. The active region is located at $y=-0.25-\mu \mathrm{m}$ below the $0 x$ axis, and the etch depth is $h=1 \mu \mathrm{m}$. A perturbation can be observed, giving a negative contribution under the stripe region, accompanied by two positive side profiles. For comparison, the case of the half-plane is presented. A larger magnitude of the antiguiding is found for the sharp profile [case a in Fig. 1(c)] and decreases if the ridge has a less steep shape. The amplitude of this effect on $\Delta n_{\mathrm{eff}}$ may be as large as about $1 \times 10^{-3}$. If the profile is steeper, the positive waveguiding side features become stronger than the magnitude of the antiguiding. The reverse is true for the less steep profile. To give some insight into the cause of this behavior, Fig. 2(c) and (d) show the dependence of the stresses $\sigma_{x x}$ and $\sigma_{y y}$ on the lateral coordinate. For the TE mode, the influence of the strain $e_{x x}$ on the changes in the refractive index is more important, so that the profile of the stress $\sigma_{x x}$ dependence almost reproduces the dependence of the refractive index change. Besides, for steeper ridge shapes, the stress $\sigma_{y y}$ contributes most to the positive waveguiding side features and 


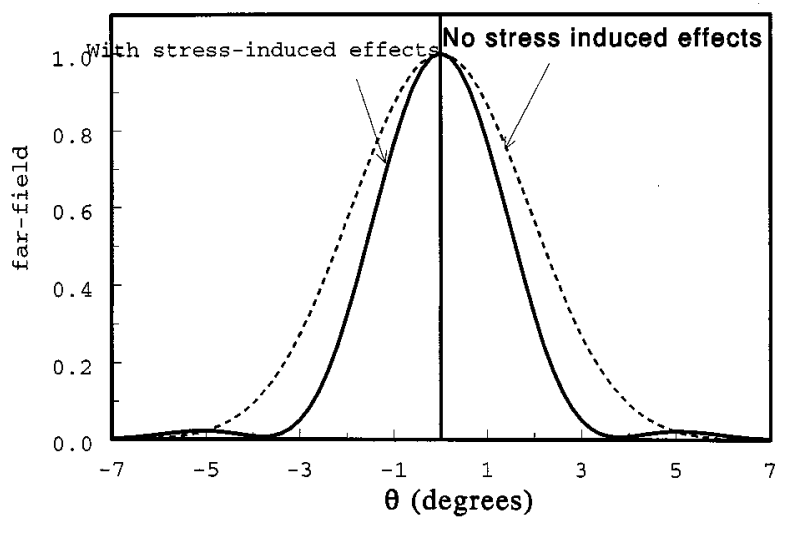

Fig. 3. Lateral far-field profile for 8 - $\mu \mathrm{m}$ wide stripe, weakly index-guided devices, with and without stress-induced effects.

may eventually lead to side lobes in the far field behavior, as will be shown below.

The magnitude of the negative variation of the refractive index under the stripe is comparable to the value corresponding to half-plane problem when the active region is placed $0.25-\mu \mathrm{m}$ below the $0 x$ axis. However, the waveguiding under the stripe region is much reduced. The amplitude of the variations near the stripe edges for the ridge case strongly depend on the steepness of the ridge profile.

We introduced these perturbations of the effective refractive index into the quasi-2-D model for the lateral behavior presented in [1], taking a weak built-in value of the index-guiding $\Delta n_{\mathrm{eff}}=4 \times 10^{-4}$ and a superimposed thermal waveguiding corresponding to a relatively large value of the threshold current density of $1800 \mathrm{~A} / \mathrm{cm}^{2}$. The value of the antiguiding factor used in this computation was $b=2$. Computing the far-field behavior at threshold, taking into account the stress-induced effect, we obtain the curve presented in Fig. 3. This shows a main lobe and two weak side lobes. These two side lobes are caused by the positive perturbations that occur because of the stress-induced effects at the stripe edges, and are observed experimentally for weakly index guided devices, as will be shown in the following section. They cannot be attributed to antiguiding since the far fields corresponding to strong antiguiding are either broader or double lobed, but without a main central lobe, and associated with larger modal losses [11].

\section{EXPERIMENTAL}

\section{A. Evaluation of the Stress in the Anodic Oxide Layer}

For evaluating the stress in the anodic oxide, we measured the radius of curvature $\rho$ of a $122-\mu$ m thick GaAs substrate covered with a $0.2-\mu \mathrm{m}$ thick anodic oxide grown under constant current conditions with a current density of $5.7 \mathrm{~mA} / \mathrm{cm}^{2}$ until the final voltage was $150 \mathrm{~V}$. After the formation of the anodic oxide, the sample was heat-treated at $400{ }^{\circ} \mathrm{C}$ for one minute. In real ridge-type devices, the anodic oxide corresponds to $\mathrm{Al}_{x} \mathrm{Ga}_{1-x} \mathrm{As}$ oxidized layers and not to GaAs; nevertheless, we use the parameters corresponding to GaAs oxide for a qual-

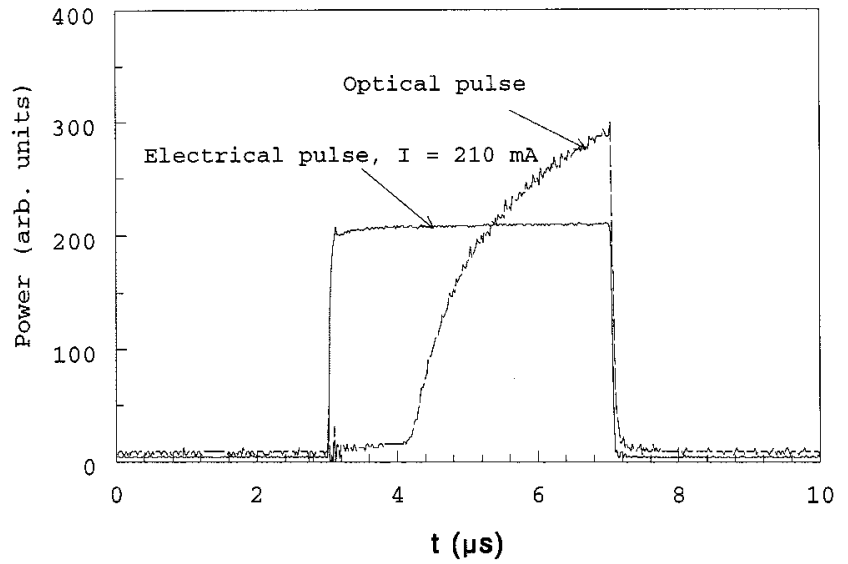

Fig. 4. Optical pulse shape at threshold $(I=210 \mathrm{~mA})$ for $8-\mu \mathrm{m}$ stripe width, 1.5 -mm long, very weakly index-guided devices.

itative estimate. If the oxide film has a thickness $t$ and is under compressive stress $\sigma$, then we find the stress from [2] to be

$$
\sigma=\frac{E \cdot d^{2}}{6 \cdot \rho \cdot(1-v)}
$$

where $E_{e} /(1-\nu)=1.23 \times 10^{11} \mathrm{dyn} / \mathrm{cm}^{2}$ in the $\{100\}$ plane of GaAs, $d$ is the thickness of the substrate, $E_{e}$ is the Young modulus, and $\nu$ is Poisson's ratio. For the specified anodic oxide, we find a compressive stress of $-3 \times 10^{9} \mathrm{dyn} / \mathrm{cm}^{2}$. This stress compares with a value of $-3 \times 10^{9} \mathrm{dyn} / \mathrm{cm}^{2}$ for thermally grown oxide films on silicon and with values in the range of $-5 \times 10^{9}$ to $-10^{10} \mathrm{dyn} / \mathrm{cm}^{2}$ for $\mathrm{SiO}_{2}: \mathrm{Si}_{3} \mathrm{~N}_{4}$ deposited on $\mathrm{GaAs}$ [2]. Also, for comparison, the value of the stress produced by the heterostructure interface mismatch should be mentioned. This is in the range of $0-1 \times 10^{8} \mathrm{dyn} / \mathrm{cm}^{2}$ [2].

\section{B. Far Field Behavior of $w=8-\mu m$ Wide Stripe Devices}

These devices have a low confinement GaAs/AlGaAs DQW asymmetric structure. Each has two 8-nm GaAs quantum wells, and an optical trap layer with an Al content of $x=0.20$ on the $\mathrm{n}$-side of the structure. The confinement factor is $7.5 \times 10^{-3}$ per QW. The device length is $1.5 \mathrm{~mm}$. The threshold current density is relatively large, i.e., $1800 \mathrm{~A} / \mathrm{cm}^{2}$, with no correction for current spreading under the stripe width.

The magnitude of the carrier-induced antiguiding is

$$
\Delta n_{\text {carrier antiguiding }}=-((b \cdot \Gamma \cdot \Delta g) /(4 \cdot \pi)) \cdot \lambda_{\circ}
$$

where

$b \quad$ antiguiding factor;

$\Gamma \quad$ confinement factor;

$g$ gain in the active region;

$\lambda_{0} \quad$ lasing wavelength (see also [1, Ch. 2]).

If we assume losses of about $3000 \mathrm{~cm}^{-1}$ outside the stripe region, in unpumped areas, and gain values of about $500 \mathrm{~cm}^{-1}$ per QW in the stripe region, we estimate the value of the carrier-induced antiguiding to be about $3 \times 10^{-4}$. In this case, the stress-induced antiguiding is larger than the corresponding carrier-induced effect, since our structures have a lower confinement factor in the active region. The built-in induced index- 

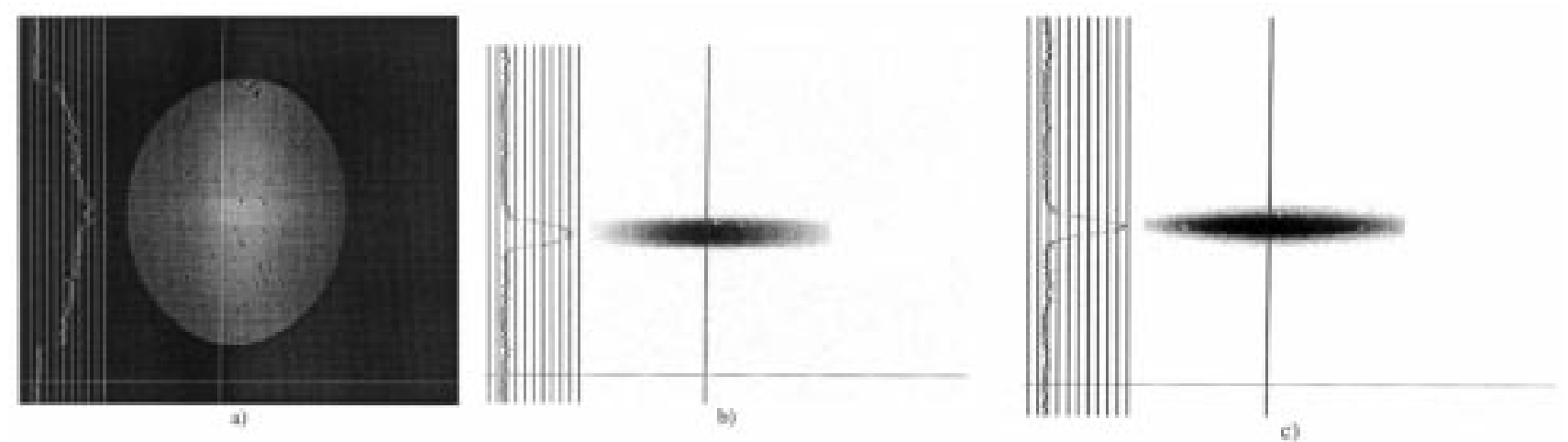

Fig. 5. (a) CW far field in the spontaneous emission, below threshold $(I=140 \mathrm{~mA})$. (b) Far field for short pulses $(300 \mathrm{~ns} / 10 \mathrm{~ms})$, above threshold $(I=280$ $\mathrm{mA})$. (c) CW far field above threshold $(I=280 \mathrm{~mA})$.

guiding was only $3-6 \times 10^{-4}$ for these devices, which is a very low value.

This is confirmed indirectly by Fig. 4, where the shape of the optical pulse is presented. We notice a large delay at threshold between the beginning of the electrical pulse and the onset of lasing. This is the clear mark of thermal effects, as presented in [1, Ch. 6]. This means that the built-in index guiding is not enough to provide waveguiding in the lateral direction and lasing has to wait until the thermally induced profile of the effective refractive index builds-up and adds to initial index-guiding. The estimated value of the thermal waveguide is in the range of $10^{-3}$, as deduced in [1]. This conclusion is also supported by the far field profiles under pulsed operation, above threshold. For a $300 \mathrm{~ns} / 10 \mathrm{~ms}$ pulse width/repetition rate and an injected current of $280 \mathrm{~mA}$, the far field, presented in Fig. 5(b), is broadened and has the characteristic shape for strong antiguiding associated with higher lateral losses [11]. In the optical pulse shape above threshold, this is observed as a rising front of the optical response, with a time constant of about $1 \mu \mathrm{s}$. When the pulse width increases more than a few microseconds, thermal effects contribute to proper waveguiding and thus the far field narrows Fig. 5(c) and the optical pulse amplitude remains constant (Fig. 4).

Stress-induced behavior is proved by Fig. 5(a) and (c). First, Fig. 5(a) presents the far field below threshold for an injected current of $140 \mathrm{~mA}$. The threshold current is about $210 \mathrm{~mA}$. Two weak side features appear, which can be correlated with perturbations at the stripe edges. Fig. 5(c) presents the far field in CW regime, which is basically the same as the far field measured under pulsed conditions if pulses are larger than a few microseconds, i.e., after the thermal waveguide builds up. Although due to the high sensitivity of the CCD camera and the quality of the graphic file, it is not clearly seen in the figure, the two weak side lobes can each be found at approximately $4.5^{\circ}$ from the normal. The width of the main lobe is about $3.7^{0}$ (full width-half amplitude). This is in qualitatively good agreement with the far field predicted theoretically in Fig. 3, in the above section.

We conclude from this that stress-induced effects are larger than carrier induced antiguiding in our low confinement structure devices and, for the given configuration of the ridge and for the given oxide, are of the order of $8 \times 10^{-4}$. Together with thermal effects, they put a lower limit on the value of the built-in index-guiding to be provided technologically in order to obtain a stable beam at higher power. The smallest possible values of the oxide thickness and a more systematic technological optimization of the oxide deposition process (considering also other type of insulators) might considerably reduce stress-induced effects. At the same time, if this effect is properly understood by further research, it may be used to provide positive waveguiding instead of antiguiding beneath the stripe region, using special layers with tensile and not compressive stress. This can be achieved using metals, for example.

\section{CONCLUSION}

Stress-induced effects can be larger than carrier-induced antiguiding in low-confinement laser diodes and may be as large as $\Delta n_{\mathrm{ef}} \approx 1 \times 10^{-3}$ for a given ridge shape and if a $0.2-\mu \mathrm{m}$ thick anodic oxide is used for electrical isolation outside the stripe region. They are manifest as antiguiding under the stripe region and of two side waveguiding features at the stripe edges. The amplitude of these effects depends also on the shape of the ridge: the steeper the shape, the larger the amplitude. Together with thermal effects, they put a lower limit on the value of the build-in index-guiding to be provided technologically in order to obtain a stable beam at higher power. In the far field, two weak side lobes are observed in the lasing regime.

Thinner oxide layers and more systematic technological optimization of the oxide deposition process (considering also other type of insulators) might considerably reduce stress-induced effects. At the same time, if this effect is properly understood by further research, it may be used to provide positive waveguiding instead of antiguiding beneath the stripe region, using special layers with tensile and not compressive stress. This can be achieved using, for example, metals.

\section{APPENDIX}

STRESS AND STRAIN COMPUTATION FOR THE HALF-PLANE AND FOR A RIDGE-SHAPED INTERFACE

\section{A. Complex Representation of the Stress Equations for the Plane Theory of Elasticity}

It is shown in [10, p. 114] that two holomorphic functions $\Phi(z), \Psi(z)$ may be used to respresent biharmonic functions involved in the theory of plane elasticity. Here, $z$ is the complex 
representation of a specific point having the plane coordinates $(x, y): z=x+i y$. If the functions $\Phi(z), \Psi(z)$ are known, the stresses are determined by

$$
\begin{gathered}
\sigma_{x x}(x, y)+\sigma_{y y}(x, y)=4 \operatorname{Re}(\Phi(z))=2(\Phi(z)+\overline{\Phi(z)}) \\
\sigma_{y y}(x, y)-\sigma_{x x}(x, y)+2 i \sigma_{x y}(x, y)=2\left(\bar{z} \cdot \Phi^{\prime}(z)+\Psi(z)\right)
\end{gathered}
$$

The corresponding strains are given by

$$
\begin{aligned}
& e_{x x}(x, y) \\
& \quad=\frac{1}{2 \mu} \cdot\left(\sigma_{x x}(x, y)-\frac{\lambda}{2(\lambda+\mu)} \cdot\left(\sigma_{x x}(x, y)+\sigma_{y y}(x, y)\right)\right) \\
& e_{y y}(x, y) \\
& \quad=\frac{1}{2 \mu} \cdot\left(\sigma_{y y}(x, y)-\frac{\lambda}{2(\lambda+\mu)} \cdot\left(\sigma_{x x}(x, y)+\sigma_{y y}(x, y)\right)\right) \\
& e_{x y}(x, y)=\frac{1}{2 \mu} \cdot \sigma_{x y}(x, y)
\end{aligned}
$$

where $\lambda, \mu$ are elastic constants related to the modulus of elasticity $E_{e}$ and to Poisson's ratio by

$$
E_{e}=\frac{\mu(3 \lambda+2 \mu)}{\lambda+\mu}, \nu=\frac{\lambda}{2(\lambda+\mu)} .
$$

The stress component $\sigma_{z z}$ is then given by

$$
\sigma_{z z}=\lambda\left(e_{x x}(x, y)+e_{y y}(x, y)\right) \text {. }
$$

\section{B. Solution for the Half-Plane}

The technique for finding the functions $\Phi(z)$, and $\Psi(z)$ is based on the theory of complex functions (Cauchy integrals) and is remarkably simple if the elastic medium is semi-infinite or bounded by a circle. We refer next to the case of a semi-infinite region, i.e., the elastic medium is found in the lower half-plane. The boundary is a line, extending to infinity in both directions. The stresses $\sigma_{y y}=N(t)$ and $\sigma_{x y}=T(t)$ are given on the axis $0 x$ as functions of the abscissa $t$.

The procedure in $[10$, p. 392] is extremely effective and elegant. We present it briefly in order to make easily understandable the extension to boundaries mapped by the function $z=$ $\omega(\tau)$ to the half-plane.

For the half-plane problem, the following boundary conditions may be written:

$$
\begin{aligned}
& \Phi(t)+\overline{\Phi(t)}+t \cdot \overline{\Phi^{\prime}(t)}+\overline{\Psi(t)}=N(t)-i T(t) \\
& \Phi(t)+\overline{\Phi(t)}+t \cdot \Phi^{\prime}(t)+\Psi(t)=N(t)+i T(t)
\end{aligned}
$$

Next, the properties of Cauchy integrals are used. Let $f(z)$ be a function holomorphic in the lower half-plane, with the possible exclusion of the points $a_{1}, \ldots, a_{n}$ where it may have poles with the principal parts $G_{1}(z), \ldots, G_{n}(z) \cdot G_{i}(z)(i=1 \ldots n)$, i.e., functions having the form

$$
G_{i}(z)=\frac{A_{1 i}}{z-a_{i}}+\frac{A_{2 i}}{\left(z-a_{i}\right)^{2}}+\cdots+\frac{A_{l i}}{\left(z-a_{i}\right)^{l}} .
$$

Then from [10, p. 277]

$$
\frac{1}{2 \pi i} \cdot \int_{-\infty}^{+\infty} \frac{f(t)}{t-z} \cdot d t=-f(z)+G_{1}(z)+\cdots+G_{n}(z)
$$

if $z$ belongs to the lower half-plane and

$$
\frac{1}{2 \pi i} \cdot \int_{-\infty}^{+\infty} \frac{f(t)}{t-z} \cdot d t=G_{1}(z)+\cdots+G_{n}(z)
$$

if $z$ belongs to the upper half-plane.

Let now $f(z)$ be a function holomorphic in the upper half-plane, with the possible exclusion of the points $a_{1}, \cdots, a_{n}$ where it may have poles with the principal parts $G_{1}(z), \cdots, G_{n}(z)$.

Then

$$
\frac{1}{2 \pi i} \cdot \int_{-\infty}^{+\infty} \frac{f(t)}{t-z} \cdot d t=f(z)-G_{1}(z)-\cdots-G_{n}(z) .
$$

if $z$ belongs to the upper half-plane and

$$
\frac{1}{2 \pi i} \cdot \int_{-\infty}^{+\infty} \frac{f(t)}{t-z} \cdot d t=-G_{1}(z)-\cdots-G_{n}(z)
$$

if $z$ belongs to the lower half-plane.

Proceeding as in [10] with the first equation in (4), we obtain

$$
\begin{aligned}
& \frac{1}{2 \pi i} \cdot \int_{-\infty}^{+\infty} \frac{\Phi(t)}{t-z} \cdot d t+\frac{1}{2 \pi i} \cdot \int_{-\infty}^{+\infty} \frac{\overline{\Phi(t)}}{t-z} \cdot d t+\frac{1}{2 \pi i} \cdot \int_{-\infty}^{+\infty} \frac{t \cdot \overline{\Phi^{\prime}(t)}}{t-z} \cdot d t \\
& \quad=\frac{1}{2 \pi i} \cdot \int_{-\infty}^{+\infty} \frac{N-i T}{t-z} \cdot d t
\end{aligned}
$$

where $z$ is any point in the lower half-plane.

It is difficult to predict the value of the terms which involve the complex conjugate of the function $\Phi(z)$, i.e., the second and the third term in (10), with the notable exception of the situations when the boundary is circular or consists of the $0 x$ axis of the half-plane. For the latter case, we notice that $\Phi(t)$ is the boundary value of $\Phi(z)$, which is holomorphic in the lower plane. Since on the boundary of the half plane $\bar{z}=z$, we can define the functions $\overline{\Phi(\bar{z})}$ and $z \cdot \overline{\Phi^{\prime}(\bar{z})}$, which are holomorphic in the upper plane and whose boundary values are $\overline{\Phi(t)}$ and $t \cdot \overline{\Phi^{\prime}(t)}$. Thus, the first integral has the value $-\Phi(z)$ while the second and the third integrals in (10) vanish. We thus obtain the function $\Phi(z)$

$$
\Phi(z)=-\frac{1}{2 \pi i} \cdot \int_{-\infty}^{+\infty} \frac{N-i T}{t-z} \cdot d t
$$

Performing a similar manipulation in the second equation in (4), we obtain the function $\Psi(z)$

$$
\Psi(z)=-\frac{1}{2 \pi i} \cdot \int_{-\infty}^{+\infty} \frac{N+i T}{t-z} \cdot d t-\Phi(z)-z \Phi^{\prime}(z) .
$$


In this way, both functions $\Phi(z)$ and $\Psi(z)$ are determined if we know the stress distribution at the boundary. Next, stress and strain functions in the lower half-plane are very easily computed as in (1) and (2).

Let us apply these formulae to the case depicted in Fig. 1, where we have two concentrated forces per unit length, i.e., $+F_{0}=\sigma_{o x} d_{o x}$ applied at $x_{1}=-W_{f} / 2$ and $-F_{0}$ applied at $x_{2}=W_{f} / 2$. Here, $\sigma_{o x}$ is the compressive stress in the oxide and $d_{o x}$ is its thickness. We can consider a constant force per unit length $F_{0}$ as being applied to a finite width $a$ and, thus, $T(t)$ is equal to $T_{0}=F_{0} / a$ for $-(a / 2) \leq t \leq(a / 2)$ and zero at rest. $N(t)$ is zero everywhere. We apply the formulae presented above even if $T(t)$ is not continuous. Similar to [1, p. 396], the correctness of the final result may be verified directly or by comparison with results presented in [2], where all formulae were obtained using another method.

If we take $T_{0} a \rightarrow \sigma_{o x} d_{o x}$ when $a \rightarrow 0$, we have two $\delta$ functions, the integral becomes $\int_{-\infty}^{+\infty} T(t) \cdot f(t) \cdot d t=-\sigma_{o x} d_{o x}$. $f\left(W_{f} / 2\right)+\sigma_{o x} d_{o x} \cdot f\left(-W_{f} / 2\right)$, and the final result takes the simple form

$$
\Phi(z)=\frac{\sigma_{o x} d_{o x}}{2 \pi} \cdot \frac{W_{f}}{z^{2}-\frac{W_{f}^{2}}{4}} .
$$

Correspondingly, $\Psi(z)$ is given by

$$
\Psi(z)=-2 \Phi(z)-z \Phi^{\prime}(z) .
$$

Thus, using (13), (14), and (1), the results in [2] are reproduced with remarkable simplicity.

\section{Solution for the Ridge Shape Mapped to the Half-Plane by the Conformal Transformation $z=\omega(\tau)$}

The powerful analysis using complex representation of stress functions allows for solving analytically the ridge shape problem if the stresses are known on the ridge boundary and if the ridge shape is mapped by a conformal transformation to the half-plane.

1) Conformal Mapping of the Ridge Shape to the Half-Plane: A reasonable approximation of the ridge shape in Fig. 2 may be obtained if we use the transformation

$$
z=\omega(\tau)=\tau-\frac{h}{2 \operatorname{arctg}\left(\frac{W}{2 p}\right)} \cdot \ln \left(\frac{\tau-\frac{W}{2}-i p}{\tau+\frac{W}{2}-i p}\right)
$$

where $\tau=\xi+i \eta$ is a point in the half-plane and $z=x+i y$ is the corresponding point in the plane of the ridge. The point corresponding to the origin $\tau=(0,0)$ is $z=(0, h)$, where $h$ is the ridge height. The parameter $p$ is related with the slope of the ridge and $W$ with its width.

Since analytical solutions are possible only for rational functions, we use the expansion

$$
z=\omega(\tau)=\tau+\frac{h}{c} \cdot \sum_{k=-2^{n-1}}^{2^{n-1}} \frac{1}{\tau-i p+\frac{W \cdot k}{2^{n}}}
$$

where the constant $c$ is determined by the condition $z=(0, h)=\omega(0,0)$. Normal shapes of the ridge can be satisfactorily described with $n \leq 5$. It is important to notice that the transformation $z=\omega(\tau)$ is holomorphic in the lower half-plane and has the poles $\tau_{k}=i p-\left((W \cdot k) /\left(2^{n}\right)\right)$ and $-2^{n-1} \leq \kappa \leq 2^{n-1}$ in the upper plane. Expressions of $\omega^{\prime}(\tau)$ and $\omega^{\prime \prime}(\tau)$ are easily found. Boundary conditions (4) become

$$
\begin{aligned}
& \Phi(s)+\overline{\Phi(s)}+\frac{1}{\omega^{\prime}(s)} \cdot\left(\omega(s) \cdot \overline{\Phi^{\prime}(s)}+\overline{\omega^{\prime}(s)} \cdot \overline{\Psi(s)}\right) \\
& =N(s)-i T(s) \\
& \Phi(s)+\overline{\Phi(s)}+\frac{1}{\overline{\omega^{\prime}(s)}}\left(\overline{\omega(s)} \cdot \Phi^{\prime}(s)+\omega^{\prime}(s) \cdot \Psi(s)\right) \\
& \quad=N(s)+i T(s)
\end{aligned}
$$

where $s$ is the line corresponding to $\eta=0(-\infty<\xi<\infty)$ in the $\tau$ half-plane. To be able to separate the function $\Phi(\tau)$ using Cauchy integrals from the first equation in (17), we multiply it by $\omega^{\prime}(s)$, and thus have

$$
\begin{aligned}
& \omega^{\prime}(s) \cdot \Phi(s)+\omega^{\prime}(s) \cdot \overline{\Phi(s)}+\omega(s) \cdot \overline{\Phi^{\prime}(s)}+\overline{\omega^{\prime}(s)} \cdot \overline{\Psi(s)} \\
& \quad=\omega^{\prime}(s) \cdot(N(s)-i T(s)) \\
& \overline{\omega^{\prime}(s)} \cdot \Phi(s)+\overline{\omega^{\prime}(s) \cdot \Phi(s)}+\overline{\omega(s)} \cdot \Phi^{\prime}(s)+\omega^{\prime}(s) \cdot \Psi(s) \\
& \quad=\overline{\omega^{\prime}(s)} \cdot(N(s)+i T(s)) .
\end{aligned}
$$

We then obtain

$$
\begin{aligned}
-\omega^{\prime}(\tau) & \cdot \Phi(\tau)+\frac{1}{2 \pi i} \cdot \int_{-\infty}^{+\infty} \frac{\omega^{\prime}(s) \cdot \overline{\Phi(s)}}{s-\tau} d s+\frac{1}{2 \pi i} \\
& +\int_{-\infty}^{+\infty} \frac{\omega(s) \cdot \overline{\Phi^{\prime}(s)}}{s-\tau} \cdot d s=\frac{1}{2 \pi i} \cdot \int_{-\infty}^{+\infty} \frac{\omega^{\prime}(s) \cdot(N-i T)}{s-\tau} \cdot d s
\end{aligned}
$$

if $\tau$ is in the lower half-plane. In deducing (19), we keep in mind that the function $\overline{\omega^{\prime}(s)} \cdot \overline{\Psi(s)}$ is the boundary value of the function $\overline{\omega^{\prime}(\bar{\tau})} \cdot \overline{\Psi(\bar{\tau})}$, which is holomorphic in the upper half-plane, and thus the Cauchy integral

$$
\frac{1}{2 \pi i} \cdot \int_{-\infty}^{+\infty} \frac{\overline{\omega^{\prime}(s)} \cdot \overline{\Psi(s)}}{s-\eta} \cdot d s
$$

is zero if $\tau=\xi+i \eta$ belongs to the lower half-plane. We have to then evaluate the second and the third term in (19). The functions $\omega^{\prime}(\tau) \cdot \overline{\Phi(\tau)}$ and $\omega(\tau) \cdot \overline{\Phi^{\prime}(\tau)}$ are holomorphic in the upper half-plane, with the exception of the known poles $\tau_{k}=i p-$ $\left((W \cdot k) /\left(2^{n}\right)\right)$, with $-2^{n-1} \leq \kappa \leq 2^{n-1}$ corresponding to the poles of the function $\omega(\tau)$.

Near the points $\overline{\tau_{k}}=-i p-\left((W \cdot k) /\left(2^{n}\right)\right)$, with $-2^{n-1} \leq$ $\kappa \leq 2^{n-1}$ (in the lower half plane), we can expand the functions $\Phi(\tau)$ and $\Phi^{\prime}(\tau)$ as

$$
\begin{aligned}
\Phi(\tau) & =C_{o k}+C_{1 k} \cdot\left(\tau-\overline{\tau_{k}}\right)+C_{2 k} \cdot\left(\tau-\overline{\tau_{k}}\right)^{2}+\cdots \\
\Phi^{\prime}(\tau) & =C_{1 k}+2 C_{2 k} \cdot\left(\tau-\overline{\tau_{k}}\right)+\cdots
\end{aligned}
$$

so that we have

$$
\begin{aligned}
\overline{\Phi(\bar{\tau})} & =\overline{C_{o k}}+\overline{C_{1 k}} \cdot\left(\tau-\tau_{k}\right)+\overline{C_{2 k}} \cdot\left(\tau-\tau_{k}\right)^{2}+\cdots \\
\overline{\Phi^{\prime}(\bar{\tau})} & =\overline{C_{1 k}}+2 \overline{C_{2 k}} \cdot\left(\tau-\tau_{k}\right)+\cdots
\end{aligned}
$$


If we now refer to the explicit expression of $\omega(\tau)(16)$, according to (9), the result of

$$
\frac{1}{2 \pi i} \cdot \int_{-\infty}^{+\infty} \frac{\omega^{\prime}(s) \cdot \overline{\Phi(s)}}{s-\tau} d s+\frac{1}{2 \pi i} \cdot \int_{-\infty}^{+\infty} \frac{\omega(s) \cdot \overline{\Phi^{\prime}(s)}}{s-\tau} \cdot d s
$$

is given by

$$
\frac{h}{c} \cdot \sum_{k=-2^{n-1}}^{2^{n=1}} \frac{\overline{C_{0 k}}}{\left(\tau-\tau_{k}\right)^{2}}
$$

with $\tau$ belonging to the lower half-plane, where $C_{0 k}=\Phi\left(\overline{\tau_{k}}\right)$.

In consequence, (19) becomes

$$
\begin{array}{r}
\omega^{\prime}(\tau) \cdot \Phi(\tau)+\frac{h}{c} \cdot \sum_{k=-2^{n-1}}^{2^{n=1}} \frac{\overline{C_{0 k}}}{\left(\tau-\tau_{k}\right)^{2}} \\
=\frac{1}{2 \pi i} \cdot \int_{-\infty}^{+\infty} \frac{\omega^{\prime}(s) \cdot(N-i T)}{s-\tau} \cdot d s
\end{array}
$$

where $\tau_{k}$ belongs to the upper half-plane. We need now to evaluate the constants $C_{0 k}$. If we write (24) for each $\tau=\overline{\tau_{k}}$, with $-2^{n-1} \leq \kappa \leq 2^{n-1}$, we obtain a linear system of $2^{n}+1$ equations, which has to be solved numerically in order to get the $2^{n}+1$ needed constants. Correspondingly, after finding $\Phi(\tau)$, we can find $\Psi(\tau)$

$$
\begin{aligned}
&-\omega^{\prime}(\tau) \cdot \Psi(\tau)+\frac{1}{2 \pi i} \cdot \int_{-\infty}^{+\infty} \frac{\omega^{\prime}(s) \cdot \overline{\Phi(s)}}{s-\tau} d s+\frac{1}{2 \pi i} \\
& \quad \cdot \int_{-\infty}^{+\infty} \frac{\omega(s) \cdot \overline{\Phi^{\prime}(s)}}{s-\tau} \cdot d s=\frac{1}{2 \pi i} \cdot \int_{-\infty}^{+\infty} \frac{\overline{\omega^{\prime}(s)} \cdot(N+i T)}{s-\tau} \cdot d s .
\end{aligned}
$$

If we apply now (6), considering that the functions $\overline{\omega^{\prime}(\tau)} \cdot \Phi(\tau)$ and $\overline{\omega(\tau)} \cdot \Phi^{\prime}(\tau)$ are holomorphic in the lower half-plane, with the exception of the known poles $\overline{\tau_{k}}=-i p-\left((W \cdot k) /\left(2^{n}\right)\right)$, $-2^{n-1} \leq \kappa \leq 2^{n-1}$ corresponding to the poles of the function $\overline{\omega(\tau)}$, we obtain:

$$
\begin{gathered}
-\omega^{\prime}(\tau) \cdot \Psi(\tau)-\overline{\omega^{\prime}(\tau)} \cdot \Phi(\tau)-\overline{\omega(\tau)} \cdot \Phi^{\prime}(\tau)-\frac{h}{\bar{c}} \cdot \sum_{k=-2^{n-1}}^{2^{n=1}} \\
\frac{C_{0 k}}{\left(\tau-\overline{\tau_{k}}\right)^{2}}=\frac{1}{2 \pi i} \cdot \int_{-\infty}^{+\infty} \frac{\omega^{\prime}(s) \cdot(N+i T)}{s-\tau} \cdot d s
\end{gathered}
$$

where $\tau$ is in the lower half-plane. Examining (26), we notice that indeed, the function $\omega^{\prime}(\tau) \Psi(\tau)$ does not have poles in the lower half-plane since the expression

$$
-\overline{\omega^{\prime}(\tau)} \cdot \Phi(\tau)-\overline{\omega(\tau)} \cdot \Phi^{\prime}(\tau)-\frac{h}{\bar{c}} \cdot \sum_{k=-2^{n-1}}^{2^{n=1}} \frac{C_{0 k}}{\left(\tau-\overline{\tau_{k}}\right)^{2}}
$$

where $\tau$ is in the neighbor of $\overline{\tau_{k}}$ cancels the term $-(h / \bar{c})$. $\sum_{k=-2^{n-1}}^{2^{n=1}}\left(C_{0 k} /\left(\tau-\overline{\tau_{k}}\right)^{2}\right)$, which goes to infinity when $\tau \rightarrow$ $\overline{\tau_{k}}$.

It remains now to find the explicit form of the term on the right side of (19), (26) which depend on the specific distribu- tion of stresses. If we have two concentrated forces (two delta functions) applied in the $\tau$ plane at $\tau_{1}=\left(-\left(W_{f} / 2\right), 0\right)$ and $\tau_{2}=\left(\left(W_{f} / 2\right), 0\right)$, where $W_{f}$ may be different than $W$, we find

$$
\begin{aligned}
& -\frac{1}{2 \pi i} \cdot \int_{-\infty}^{+\infty} \frac{\omega^{\prime}(s) \cdot(N-i T)}{s-\tau} \cdot d s \\
& =\Phi_{0}(\tau) \cdot\left(1-\frac{h}{c} \cdot \sum_{k=-2^{n-1}}^{2^{n-1}} \frac{\frac{W_{f}^{2}}{4}+{\overline{\tau_{k}}}^{2}-2 \cdot \tau \cdot \overline{\tau_{k}}}{\left(\frac{W_{f}^{2}}{4}-{\overline{\tau_{k}}}^{2}\right)^{2}}\right)
\end{aligned}
$$

and respectively

$$
\begin{aligned}
& \frac{1}{2 \pi i} \cdot \int_{-\infty}^{+\infty} \frac{\overline{\omega^{\prime}(s)} \cdot(N+i T)}{s-\tau} \cdot d s \\
& \quad=\Phi_{0}(\tau) \cdot\left(1-\frac{h}{\bar{c}} \cdot \sum_{k=-2^{n-1}}^{2^{n-1}} \frac{\frac{W_{f}^{2}}{4}+{\overline{\tau_{k}}}^{2}+2 \cdot \tau \cdot \overline{\tau_{k}}}{\left(\frac{W_{f}^{2}}{4}-{\overline{\tau_{k}}}^{2}\right)^{2}}\right)
\end{aligned}
$$

where

$$
\Phi_{o}(\tau)=\frac{\sigma_{o x} d_{o x}}{2 \pi} \cdot \frac{W_{f}}{\tau^{2}-\frac{W_{f}^{2}}{4}}
$$

is the function $\Phi(\tau)$ for the half-plane, as in (13).

As shown in [10, p. 14], the solution for concentrated forces is completely defined only if there is a definite limiting process. In our case, we have a force $F_{0}$ being applied to a finite width $a$, and thus $T(t)$ equals $-T_{0}=-F_{0} / a$ for

$$
-\frac{a}{2}+\frac{W}{2} \leq t \leq \frac{a}{2}+\frac{W}{2}
$$

and equals $T_{0}=F_{0} / a$ for

$$
-\frac{a}{2}-\frac{W}{2} \leq t \leq \frac{a}{2}-\frac{W}{2}
$$

and zero in rest, as explained above. $N(t)$ is zero everywhere. Similarly, if we evaluate the right side of (19) and (26) for this finite case, and then go to the limit, it is easy to check that the solution corresponding to $a \rightarrow 0$ is the same as the one presented above using delta functions. For the sake of completeness, we present this case

$$
\begin{aligned}
& -\frac{1}{2 \pi i} \cdot \int_{-\infty}^{+\infty} \frac{\omega^{\prime}(s) \cdot(N-i T)}{s-\tau} \cdot d s \\
& \quad=-\frac{\sigma_{0 x} \cdot d_{0 x}}{2 \pi a} \cdot\left[\ln \left(\frac{\left(\frac{W_{f}+a}{2}\right)^{2}-\tau^{2}}{\left(\frac{W_{f}-a}{2}\right)^{2}-\tau^{2}}\right)-\frac{h}{c} \cdot \sum_{k=-2^{n-1}}^{2^{n-1}} I_{k a}\right]
\end{aligned}
$$


and respectively

$$
\begin{aligned}
& \frac{1}{2 \pi i} \cdot \int_{-\infty}^{+\infty} \frac{\overline{\omega^{\prime}(s)} \cdot(N+i T)}{s-\tau} \cdot d s \\
& =-\frac{\sigma_{0 x} \cdot d_{0 x}}{2 \pi a} \cdot\left[\ln \left(\frac{\left(\frac{W_{f}+a}{2}\right)^{2}-\tau^{2}}{\left(\frac{W_{f}-a}{2}\right)^{2}-\tau^{2}}\right)-\frac{h}{\bar{c}} \cdot \sum_{k=-2^{n-1}}^{2^{n-1}} I_{k b}\right]
\end{aligned}
$$

where

$$
\begin{aligned}
I_{k a}= & \frac{1}{\left(\tau_{k}-\tau\right)^{2}} \\
& \cdot \ln \left\{\frac{\left[\left(\frac{W_{f}+a}{2}\right)^{2}-\tau^{2}\right] \cdot\left[\left(\frac{W_{f}-a}{2}\right)^{2}-\tau_{k}^{2}\right]}{\left[\left(\frac{W_{f}-a}{2}\right)^{2}-\tau^{2}\right] \cdot\left[\left(\frac{W_{f}+a}{2}\right)^{2}-\tau_{k}^{2}\right]}\right\} \\
& +\frac{a}{\left(\tau_{k}-\tau\right)} \cdot \frac{2 \cdot W_{f} \cdot \tau_{k}}{\left[\left(\frac{W_{f}-a}{2}\right)^{2}-\tau_{k}^{2}\right] \cdot\left[\left(\frac{W_{f}+a}{2}\right)^{2}-\tau_{k}^{2}\right]}
\end{aligned}
$$

and respectively

$$
\begin{aligned}
I_{k b}= & \frac{1}{\left(\overline{\tau_{k}}-\tau\right)^{2}} \\
& \cdot \ln \left\{\frac{\left[\left(\frac{W_{f}+a}{2}\right)^{2}-\tau^{2}\right] \cdot\left[\left(\frac{W_{f}-a}{2}\right)^{2}-\bar{\tau}^{2}\right]}{\left[\left(\frac{W_{f}-a}{2}\right)^{2}-\tau^{2}\right] \cdot\left[\left(\frac{W_{f}+a}{2}\right)^{2}-\bar{\tau}_{k}^{2}\right]}\right\} \\
& +\frac{a}{\left(\overline{\tau_{k}}-\tau\right)} \cdot \frac{2 \cdot W_{f} \cdot \bar{\tau}_{k}}{\left[\left(\frac{W_{f}-a}{2}\right)^{2}-\bar{\tau}_{k}^{2}\right] \cdot\left[\left(\frac{W_{f}+a}{2}\right)^{2}-\overline{\tau_{k}^{2}}\right] .}
\end{aligned}
$$

If the functions $\Phi(\tau)$ and $\Psi(\tau)$ for all points corresponding to the lower half-plane are found, and then in the found solution, we put again $\tau=(\xi, 0)$, we must obtain the imposed values on the boundary. It is important to notice that, in this way, we can check the correctness of the solution, also in the computer program, which calculates the expressions above.

After finding the functions $\Phi(\tau)$ and $\Psi(\tau)$, the problem is solved. The stresses $\sigma_{x x}(x, y), \sigma_{y y}(x, y), \sigma_{x y}(x, y)$ in the $z=0$ plane, i.e., $(x, y)$ coordinates, are given by

$$
\begin{aligned}
& \sigma_{x x}(x, y)+\sigma_{y y}(x, y)=2 \operatorname{Re}(\Phi(z)) \\
& \sigma_{y y}(x, y)-\sigma_{x x}(x, y)+2 i \cdot \sigma_{x y}(x, y) \\
& \quad=\frac{2}{\omega^{\prime}(z)} \cdot\left[\overline{\omega(z)} \cdot \Phi^{\prime}(z)+\omega^{\prime}(z) \cdot \Psi(z)\right]
\end{aligned}
$$

and the corresponding strain functions are easily obtained as in (2). Here we are mainly interested in finding stress and strain functions at the location of the active region, i.e., at a certain depth below the boundary.

For the $\tau$ plane, i.e., in the curvilinear $(\xi, \eta)$ coordinates, similar relationships as (1) hold

$$
\begin{aligned}
& \sigma_{\xi \xi}(\xi, \eta)+\sigma_{\eta \eta}(\xi, \eta)=2 \operatorname{Re}(\Phi(\tau)) \\
& \sigma_{\eta \eta}(\xi, \eta)-\sigma_{\xi \xi}(\xi, \eta)+2 i \cdot \sigma_{\xi \eta}(\xi, \eta) \\
& \quad=\frac{2}{\overline{\omega^{\prime}(\tau)}} \cdot\left[\overline{\omega(\tau)} \cdot \Phi^{\prime}(\tau)+\omega^{\prime}(\tau) \cdot \Psi(\tau)\right]
\end{aligned}
$$

The latter formulae may be used to check the correctness of the solution, as described above. That means that we must obtain for points on the $0 \xi$ axis $(\eta=0)$, a stress function $\sigma_{\xi \eta}(\xi)$, which equals $-T_{0}=-F_{0} / a$ for

$$
-\frac{a}{2}+\frac{W_{f}}{2} \leq t \leq \frac{a}{2}+\frac{W_{f}}{2}
$$

and $T_{0}=F_{0} / a$ for

$$
-\frac{a}{2}-\frac{W_{f}}{2} \leq t \leq \frac{a}{2}-\frac{W_{f}}{2}
$$

The stress function $\sigma_{\eta \eta}(\xi)$ must be zero everywhere on the boundary.

Alternatively, for points in the lower half-plane, we can compare the computed solution above in the limit of $a \rightarrow 0$ with the solution for concentrated forces, where the limit $a \rightarrow 0$ is already included within the analytic formulae.

\section{REFERENCES}

[1] M. Buda, "Low confinement high power semiconductor lasers," Ph.D. dissertation, Eindhoven University of Technology, Eindhoven, The Netherlands, 1999.

[2] P. A. Kirkby and P. R. Selway, "Photoelastic waveguides and their effect on stripe-geometry GaAs/Ga $\mathrm{Ga}_{1-x} \mathrm{Al}_{x}$ As lasers," J. Appl. Phys., vol. 50, no. 7, pp. 4567-4579, 1979.

[3] R. Maciejko, J. M. Glinski, A. Champagne, J. Berger, and L. Samson, "Photoelastic effects on the emission patterns of InGaAsP ridge-waveguide lasers," IEEE J. Quantum Electron., vol. 25, pp. 651-661, Apr. 1989.

[4] A. R. Goodwin, P. A. Kirkby, I. G. A. Davies, and R. S. Baulcomb, "The effects of processing stresses on residual degradation in long-lived $\mathrm{Ga}_{x} \mathrm{Al}_{1-x}$ As laser," Appl. Phys. Lett., vol. 34, no. 10, pp. 647-649, 1979.

[5] Q. Z. Liu, W. X. Chen, N. Y. Li, L. S. Yu, C. W. Tu, P. K. L. Yu, S. S. Lau, and H. P. Zappe, "Planar semiconductor lasers using the photoelastic effect," J. Appl. Phys., vol. 83, no. 12, pp. 7442-7447, 1998.

[6] P. Barrios and H. K. Kim, "Stress-induced lateral confinement of light in epitaxial $\mathrm{BaTiO}_{3}$ films grown by radio-frequency magnetron sputtering," Appl. Phys. Lett., vol. 73, no. 8, pp. 1017-1019, 1998.

[7] F. H. Peters and D. Cassidy, "Spatially and polarization resolved electroluminiscence of $1.3 \mu \mathrm{m}$ InGaAsP semiconductor diode lasers," Appl. Opt., vol. 28, no. 17, pp. 3744-3750, 1989.

[8] P. D. Colbourne and D. Cassidy, "Imaging of stress in GaAs diode lasers using polarization-resolved photoluminiscence," IEEE J. Quantum Electron., vol. 29, pp. 62-69, Jan. 1993.

[9] J. Yang and D. Cassidy, "Correlation between strain fields on the facet and along the cavity in semiconductor diode lasers," J. Appl. Phys., vol. 77, no. 8, pp. 3762-3765, 1995.

[10] N. Muskhelishvili, Some Basic Problems of the Mathematical Theory of Elasticity (in Russian by translated J. R. M. Radok). Gronigen, The Netherlands: Noordhoff, 1963.

[11] P. S. Zory, Ed., Quantum Well Lasers. New York: Academic, 1993. 
M. Buda was born in 1962 in Bucharest, Romania, and graduated from the Faculty of Physics, University of Bucharest, Bucharest, Hungary, in 1984.

Her research experience is related to IRED's for data fiber transmission and to high-power semiconductor laser diodes. During 1988-1997, she was with the Institute of Physics and Technology of Materials, Bucharest, Romania, as a Researcher in the Laser Diode Group. Her main interests lie in improving high-power laser diode performances using the concept of "low confinement." During 1997-1999, she was with the Electronic Devices Group, University of Eindhoven, Eindhoven, The Netherlands. In 1999, she moved to the Physics Group, working in growth and characterisation of InGaAsN for optoelectronic applications.

G. Iordache, photograph and biography not available at time of publication.

G. A. Acket, photograph and biography not available at time of publication.

T. G. van de Roer was born in Brunssum, The Netherlands, in 1937, and graduated in 1964 from the Department of Electrical Engineering, Eindhoven University of Technology, Eindhoven, The Netherlands, where he also received the Ph.D. degree in 1977.

Since 1964, he has been with Eindhoven University, presently as an Associate Professor. In 1967-1968, on a NATO fellowship, he was a Visiting Researcher in the Microwave Department, Royal Institute of Technology, Stockholm, Sweden. During 1984, he was with the Laser Technology Group, Philips Research Laboratories, Eindhoven, The Netherlands. His present interests are mainly in the modeling of a wide class of devices, including field effect transistors, double barrier resonant tuneling diodes, and diode lasers. He has co-authored over 30 papers and a book.
L. M. F. Kaufmann, received the M.S. degree in 1972 and the Ph.D. degree in 1976, both in electrical engineering, from the Rheinisch- Westfalische Tehnische Hochschule (RWTH), Aachen, Germany.

He was involved in III-V device technology, focusing on the epitaxial growth of active layers for GUNN-devices and MESFETs using modified liquid phase epitaxy (LPE) processes. Between 1976 and 1988, he was a member of the Semiconductor Devices and Technology Section, Gerhard-Mercator University, Duisburg, and was responsible for the III-V Device Technology Laboratories Since 1988, he has been appointed to the Chair of the Section Electronic Devices, Eindhoven University of Technology (EUT), Eindhoven, The Netherlands. He contributed to the development of materials, components and circuits for (opto-)electronic devices. He is co-founder of the EUT Inter-University Research Institute on Communication Technology COBRA.

B. H. van Roy, photograph and biography not available at time of publication.

E. Smalbrugge, photograph and biography not available at time of publication

I. Moerman, photograph and biography not available at time of publication.

C. Sys, photograph and biography not available at time of publication. 